RESCEU-2/99

UTAP-317/98

Advances in Space Research, in press.

\title{
COSMOLOGICAL IMPLICATIONS OF GALAXY CLUSTERS IN X-RAY, MILLIMETER, AND SUBMILLIMETER BANDS
}

\author{
Yasushi Suto ${ }^{1,2}$, Tetsu Kitayama ${ }^{2}$, Eiichiro Komatsu ${ }^{3}$, Makoto Hattori ${ }^{3}$, Ryohei Kawabe ${ }^{4}$ Hiroshi Matsuo $^{4}$, \\ Sabine Schindler ${ }^{5}$, and Kohji Yoshikawa ${ }^{6}$ \\ ${ }^{1}$ Research Center for the Early Universe, School of Science, University of Tokyo, Tokyo 113-0033, Japan \\ ${ }^{2}$ Department of Physics, University of Tokyo, Tokyo 113-0033, Japan \\ ${ }^{3}$ Astronomical Institute, Tôhoku University, Sendai 980-8578, Japan \\ ${ }^{4}$ Nobeyama Radio Observatory, Nagano 384-1305, Japan \\ ${ }^{5}$ Astrophysics Research Institute, Liverpool John Moores University, Liverpool L3 3AF, UK \\ ${ }^{6}$ Department of Astronomy, Kyoto University, Kyoto 606-8502, Japan
}

\begin{abstract}
Cosmological implications of clusters of galaxies are discussed with particular attention to their importance in probing the cosmological parameters. More specifically we compute the number counts of clusters of galaxies, $\log N-\log S$ relation, in X-ray and submm bands on the basis of the Press-Schechter theory. As an important step toward breaking the degeneracy among the viable cosmological models, we observed the most luminous X-ray cluster RXJ1347-1145 in three bands (21 and $43 \mathrm{GHz}$ in the Nobeyama Radio Observatory, Japan, and $350 \mathrm{GHz}$ in the J. C. Maxwell telescope at Mauna Kea, Hawaii). We report on the preliminary results which are in good agreement with the profile of the Sunayev - Zel'dovich effect predicted on the basis of the previous X-ray observation of the cluster.
\end{abstract}

\section{INTRODUCTION}

There are several reasons why clusters of galaxies are regarded as useful tools in cosmology; (i) since the dynamical time-scale of clusters is comparable to the age of the universe, they should retain the cosmological initial conditions fairly faithfully, (ii) clusters can be observed in various wavelengths including optical, X-ray, radio, mm and submm bands, and recent and future big projects (e.g., SDSS, AXAF, PLANCK) aim to make extensive surveys and detailed imaging/spectroscopic observations of clusters, (iii) to the first order approximation, clusters can be regarded as a simple system of dark matter, gas and galaxies, and thus theoretically well-defined and relatively well-understood, at least compared with galaxies themselves, (iv) clusters of galaxies can be observed up to high redshifts and thus provide a probe of the distant universe.

X-ray observations are particularly suited for the study of clusters, because the X-ray emissivity is proportional to $n_{e}^{2}$ and less sensitive to the projection contamination which has been known to be a serious problem in their identifications with the optical data. In addition, the Sunyaev - Zel'dovich (SZ) effect (Sunyaev \& Zel'dovich 1972), observed in radio, millimeter and submillimeter bands, is now opening a new window to investigating cluster properties, especially at high redshifts. In this paper, we aim to show the significance of current and future observations in these bands in cosmology. 


\section{LOG $N$ - LOG $S$ OF X-RAY CLUSTERS}

We compute the number of clusters observed per unit solid angle with X-ray flux greater than $S$ by

$$
N(>S)=\int_{0}^{\infty} d z d_{A}^{2}(z) c\left|\frac{d t}{d z}\right| \int_{S}^{\infty} d S(1+z)^{3} n_{M}(M, z) \frac{d M}{d T_{\text {gas }}} \frac{d T_{\text {gas }}}{d L_{\text {band }}} \frac{d L_{\text {band }}}{d S}
$$

where $c$ is the speed of light, $t$ is the cosmic time, $d_{A}$ is the angular diameter distance, $T_{\text {gas }}$ and $L_{\text {band }}$ are respectively the gas temperature and the band-limited absolute luminosity of clusters, and $n_{M}(M, z) d M$ is the comoving number density of virialized clusters of mass $M \sim M+d M$ at redshift $z$.

Given the observed flux $S$ in an X-ray energy band $\left[E_{a}, E_{b}\right]$, the source luminosity $L_{b a n d}$ at $z$ in the corresponding band $\left[E_{a}(1+z), E_{b}(1+z)\right]$ is written as

$$
L_{\text {band }}\left[E_{a}(1+z), E_{b}(1+z)\right]=4 \pi d_{L}^{2}(z) S\left[E_{a}, E_{b}\right],
$$

where $d_{L}=(1+z)^{2} d_{A}$ is the luminosity distance. We adopt the observed $L_{b o l}-T_{\text {gas }}$ relation parameterized by

$$
L_{b o l}=L_{44}\left(\frac{T_{g a s}}{6 \mathrm{keV}}\right)^{\alpha}(1+z)^{\zeta} 10^{44} h^{-2} \mathrm{erg} \mathrm{sec}^{-1} .
$$

We take $L_{44}=2.9, \alpha=3.4$ and $\zeta=0$ as a fiducial set of parameters on the basis of recent observational indications (David et al. 1993; Mushotzky \& Scharf 1997). Then we translate $L_{b o l}\left(T_{g a s}\right)$ into the bandlimited luminosity $L_{b a n d}\left[T_{g a s}, E_{1}, E_{2}\right]$ by properly taking account of metal line emissions in addition to the thermal bremsstrahlung (we fix the abundance of intracluster gas as 0.3 times the solar value).

Assuming that the intracluster gas is isothermal, its temperature $T_{\text {gas }}$ is related to the total mass $M$ by

$$
\begin{aligned}
k_{B} T_{\text {gas }} & =\gamma \frac{\mu m_{p} G M}{3 r_{\mathrm{vir}}\left(M, z_{f}\right)} \\
& =5.2 \gamma\left(1+z_{f}\right)\left(\frac{\Delta_{\mathrm{vir}}}{18 \pi^{2}}\right)^{1 / 3}\left(\frac{M}{10^{15} h^{-1} M_{\odot}}\right)^{2 / 3} \Omega_{0}^{1 / 3} \mathrm{keV} .
\end{aligned}
$$

where $k_{B}$ is the Boltzmann constant, $G$ is the gravitational constant, $m_{p}$ is the proton mass, $\mu$ is the mean molecular weight (we adopt $\mu=0.59$ ), and $\gamma$ is a fudge factor of order unity which may be calibrated from hydrodynamical simulations or observations. We adopt $\gamma=1.2$ as canonical based on the recent simulation results (e.g. Bryan \& Norman 1998). The virial radius $r_{\text {vir }}\left(M, z_{f}\right)$ of a cluster of mass $M$ virialized at $z_{f}$ is computed from $\Delta_{\text {vir }}$, the ratio of the mean cluster density to the mean density of the universe at that epoch. We evaluate this quantity using the formulae for the spherical collapse model presented in Kitayama \& Suto (1996b) and assuming for simplicity that $z_{f}$ is equal to the epoch $z$ at which the cluster is observed. Finally, we compute the mass function $n_{M}(M, z) d M$ in equation (11) using the Press-Schechter theory (Press \& Schechter 1974) assuming $z=z_{f}$ as above. The effect of $z_{f} \neq z$ is

discussed by Kitayama \& Suto (1997) in this context, and the more general consideration of $z_{f} \neq z$ is given in Lacey \& Cole (1993), Sasaki (1994), and Kitayama \& Suto (1996a,b).

\section{BREAKING THE DEGENERACY WITH THE SUNYAEV - ZEL'DOVICH EFFECT}

As has been realized for a while, a cosmological observation is often accounted for by a set of quite different models. In the present case, we found that the following models equally fit the $\log N-\log S$ of ROSAT X-ray clusters (quoted numbers are $1 \sigma$ statistical and systematic errors, respectively):

$$
\sigma_{8}=(0.54 \pm 0.02 \pm 0.08) \times \Omega_{0}^{-0.35-0.80 \Omega_{0}+0.55 \Omega_{0}^{2}}
$$


for $\lambda_{0}=1-\Omega_{0}$ Cold Dark Matter (CDM) models, and

$$
\sigma_{8}=(0.54 \pm 0.02 \pm 0.08) \times \Omega_{0}^{-0.28-0.91 \Omega_{0}+0.68 \Omega_{0}^{2}}
$$

for $\lambda_{0}=0 \mathrm{CDM}$ models. In order to exhibit this degeneracy among viable cosmological models, we select several examples listed in Table 1 which reproduce the ROSAT Log $N-\log S$ in almost an indistinguishable manner (Fig.11a).

Table 1: CDM models from the ROSAT X-ray $\log N-\log S$.

\begin{tabular}{ccccccc}
\hline Model & $\Omega_{0}$ & $\lambda_{0}$ & $h$ & $\sigma_{8}$ & $\alpha$ & $\gamma$ \\
\hline L03 & 0.3 & 0.7 & 0.7 & 1.04 & 3.4 & 1.2 \\
O045 & 0.45 & 0 & 0.7 & 0.83 & 3.4 & 1.2 \\
E1 & 1.0 & 0 & 0.5 & 0.56 & 3.4 & 1.2 \\
L03 $\gamma$ & 0.3 & 0.7 & 0.7 & 0.90 & 3.4 & 1.5 \\
L01 $\alpha$ & 0.1 & 0.9 & 0.7 & 1.47 & 2.7 & 1.2 \\
\hline
\end{tabular}

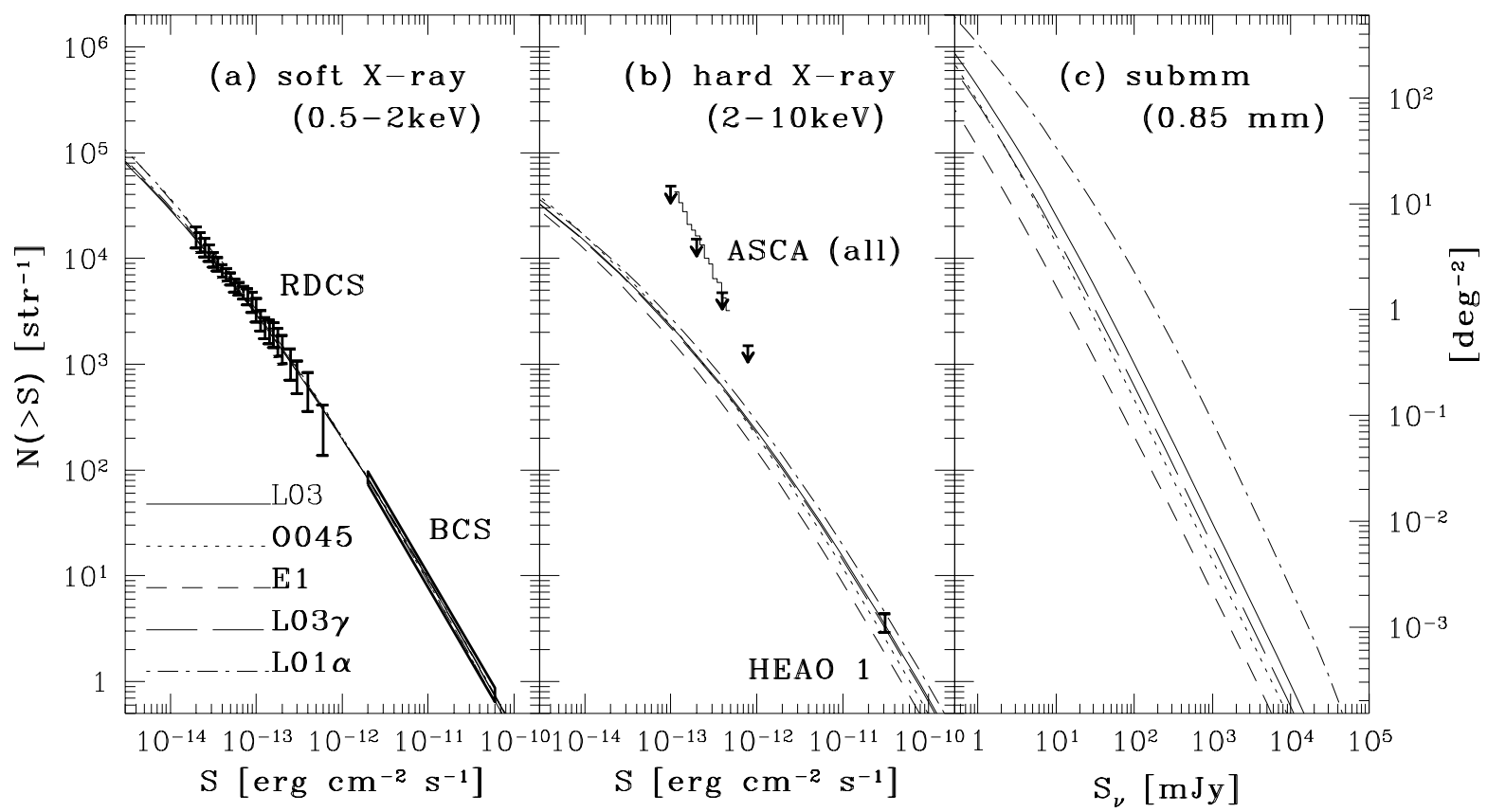

Figure 1: $\log N-\log S$ relations of galaxy clusters for CDM models in (a) the soft X-ray $(0.5-2 \mathrm{keV})$ band, (b) the hard X-ray $(2-10 \mathrm{keV})$ band, and (c) the submm $(0.85 \mathrm{~mm})$ band. Lines represent the models listed in Table 1; L03 (solid), O045 (dotted), E1 (short dashed), L03 $\gamma$ (long dashed), and L01 $\alpha$ (dotdashed). Also shown in panel (a) are the $1 \sigma$ error bars from the ROSAT Deep Cluster Survey (Rosati et al. 1995, 1997), and the error box from the ROSAT Brightest Cluster Sample (Ebeling et al. 1997, 1998). In panel (b), the number of clusters observed by $H E A O 1$ (Piccinotti et al. 1982) is indicated by the $1 \sigma$ error bar, and the counts of all X-ray sources detected by $A S C A$ are plotted for reference as a histogram (Ueda et al. 1998) and downward arrows (Cagnoni et al. 1998).

While this degeneracy can be broken by several methods, one possibility is to combine with observations of the Sunyaev \& Zel'dovich (SZ) effect, the distortion of the cosmic microwave background (CMB) spectrum due to the inverse-Compton scattering in high temperature intracluster electrons (Sunyaev \& 
Zel'dovich 1972, SZ). The specific intensity of the SZ signal at the rest frame of the cluster is given by

$$
\begin{aligned}
I_{\nu}^{\mathrm{NR}} & =\frac{2 h_{\mathrm{P}} \nu^{3}}{c^{2}} \tilde{n}=i_{0}(1+z)^{3} \frac{x^{3}}{e^{x}-1}+\Delta I_{\nu}^{\mathrm{NR}}, \\
\Delta I_{\nu}^{\mathrm{NR}} & =i_{0}(1+z)^{3} g(x) y
\end{aligned}
$$

where $h_{\mathrm{P}}$ is the Planck constant, and $y$ is the Compton $y$-parameter:

$$
y \equiv \int \frac{k_{\mathrm{B}} T}{m_{\mathrm{e}} c^{2}} \sigma_{\mathrm{T}} n_{\mathrm{e}} c d t
$$

In the above expressions, $T_{2.73}$ is the CMB temperature $T_{\gamma 0}$ divided by $2.73 \mathrm{~K}, x$ is the photon energy in units of $k T_{\gamma 0}$, and

$$
\begin{aligned}
i_{0} & =\frac{2\left(k_{\mathrm{B}} T_{\gamma 0}\right)^{3}}{\left(h_{\mathrm{P}} c\right)^{2}}=2.29 \times 10^{4} T_{2.73}^{3}{\mathrm{mJy} \operatorname{arcmin}^{-2}}^{-2} \\
g(x) & =\frac{x^{4} e^{x}}{\left(e^{x}-1\right)^{2}}\left(x \operatorname{coth} \frac{x}{2}-4\right) .
\end{aligned}
$$

By integrating equation (7) over a cluster, one obtains the excess flux from the cluster relative to the CMB:

$$
\begin{aligned}
S_{\nu}^{\mathrm{NR}} & =\frac{1}{d_{\mathrm{L}}^{2}(z)} \int \Delta I_{\nu}^{\mathrm{NR}} d A \\
& =4.9 g(x) \frac{2-Y}{2}\left[\frac{d_{\mathrm{A}}(z)}{c H_{0}^{-1}}\right]^{-2}\left(\frac{M}{10^{15} h^{-1} M_{\odot}}\right)\left(\frac{T}{\mathrm{keV}}\right) \frac{\Omega_{\mathrm{B}} h}{\Omega_{0}} \text { mJy. }
\end{aligned}
$$

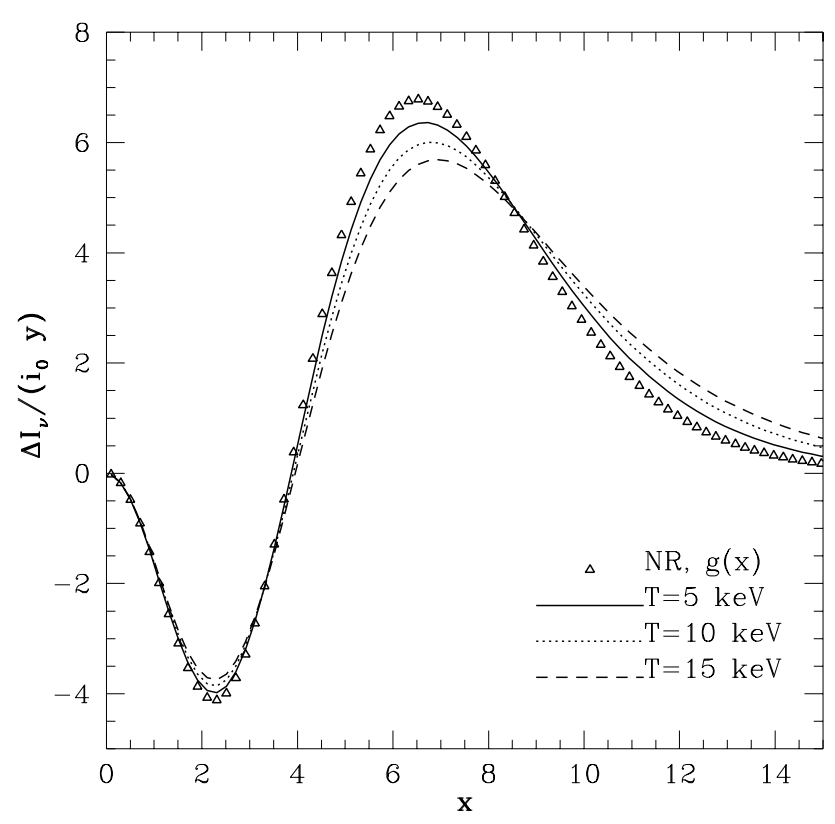

Figure 2: The spectral dependence of the intensity change $\Delta I_{\nu} /\left(i_{0} y\right)$ induced by the SZ effect. The non-relativistic expression $g(x)$ is shown as triangles, whereas the relativistic results are plotted as lines for $T=5 \mathrm{keV}$ (solid), $10 \mathrm{keV}$ (dotted), and $15 \mathrm{keV}$ (dashed).

The spectrum of the SZ effect is completely specified by the function $g(x)$ which is independent of cluster properties. Most importantly, $g(x)$ is negative at $x<3.83(\nu<217 \mathrm{GHz}, \lambda>0.14 \mathrm{~cm})$, while positive at $x>3.83$ (Fig.2). Clusters of galaxies therefore serve as negative (absorbing) sources at $\mathrm{mm}$ and $\mathrm{cm}$ bands, 
and as positive (emitting) sources at submm band. At $T \gtrsim 10 \mathrm{keV}$, relativistic effects of electrons become important and the above expressions need to be modified. We thus apply the relativistic correction to the intensity change derived by Rephaeli (1995; see also Rephaeli \& Yankovitch 1997): At the observed wavelength of $0.85 \mathrm{~mm}$, the above correction leads to $4 \%, 11 \%$ and $16 \%$ reduction in the excess flux, i.e., $g(x)$, at $T=3 \mathrm{keV}, 8 \mathrm{keV}$ and $12 \mathrm{keV}$, respectively. While clusters with $T \gtrsim 10 \mathrm{keV}$ are rare and the above correction does not make significant difference, we take it into account for completeness.

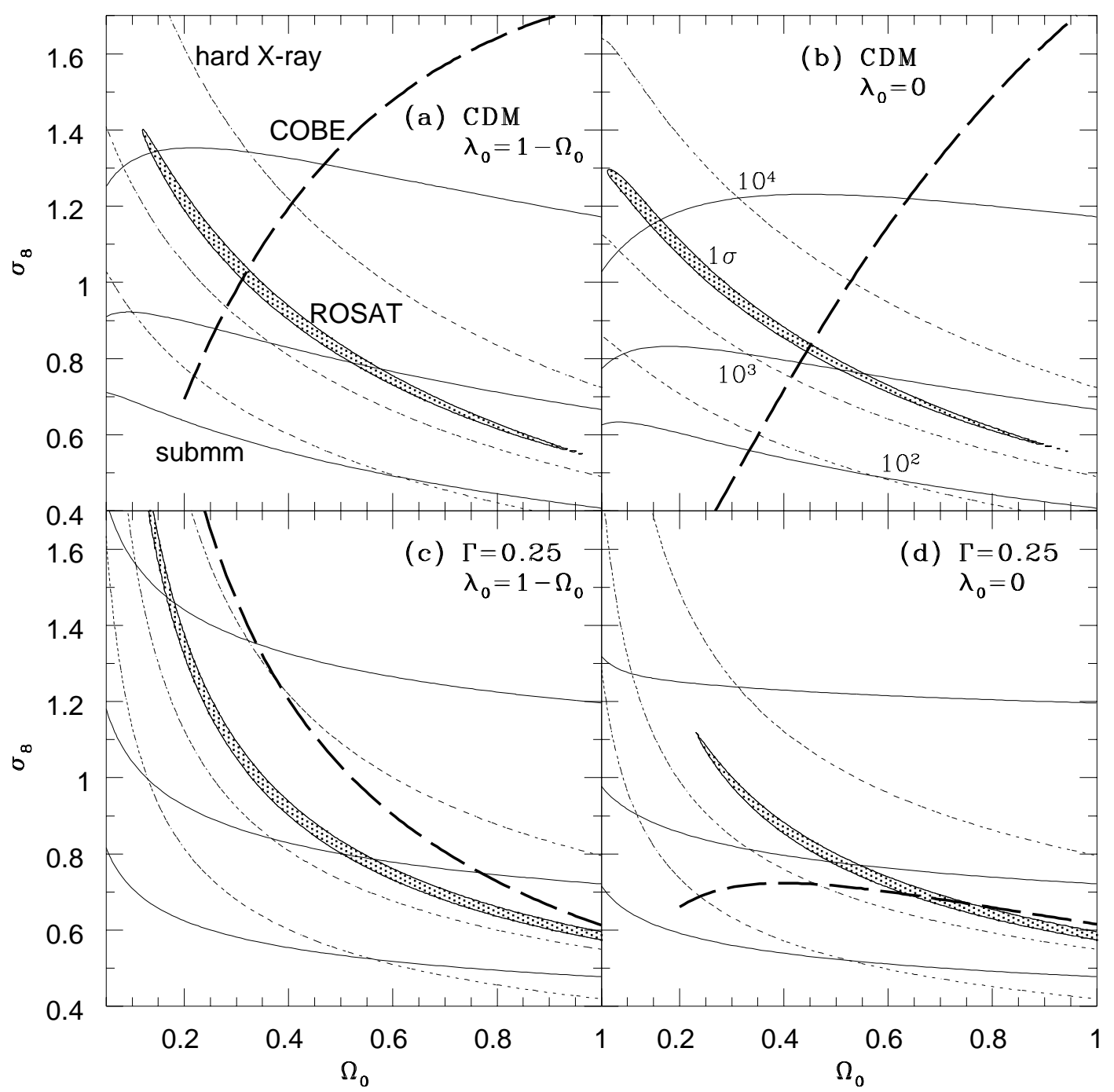

Figure 3: Contour maps on the $\Omega_{0}-\sigma_{8}$ plane in (a) spatially flat $\left(\lambda_{0}=1-\Omega_{0}\right)$ CDM models, (b) open $\left(\lambda_{0}=0\right)$ CDM models, (c) spatially flat CDM-like models with the fixed shape parameter $(\Gamma=0.25)$, and (d) open CDM-like models with $\Gamma=0.25$. In all cases, $h=0.7$ and the fiducial set of intracluster gas parameters is assumed. Shaded regions represent the $1 \sigma$ significance contours from the soft X-ray $(0.5-2 \mathrm{keV}) \log N-\log S$. Dotted and solid lines indicate the contours of the number of clusters greater than $S$ per steradian $\left(10^{2}, 10^{3}, 10^{4}\right.$ from bottom to top) with $S=10^{-13} \mathrm{erg} \mathrm{cm}^{-2} \mathrm{~s}^{-1}$ in the hard X-ray $(2-10 \mathrm{keV})$ band and with $S_{\nu}=50 \mathrm{mJy}$ in the submm $(0.85 \mathrm{~mm})$ band, respectively. Thick dashed lines represent the COBE 4 year results by Bunn \& White (1997).

Panels (b) and (c) in Figure 1 show the number counts of clusters of galaxies in the hard X-ray $(2-10$ $\mathrm{keV})$ and the submm (0.85 mm, due to the SZ effect) bands for models which reproduce the observed Log $N-\log S$ in the soft X-ray band (Table 1). Clearly the predictions in submm bands are significantly different among the models. To see this clearly, we plot in Figure 3 contour maps of the cluster $\log N-\log S$ in different bands on the $\Omega_{0}-\sigma_{8}$ plane. For the $0.5-2 \mathrm{keV}$ band, the $1 \sigma$ significance level is plotted, 
while for the $2-10 \mathrm{keV}$ and submm bands, the contours of the number of clusters per steradian $\left(10^{2}, 10^{3}\right.$, $10^{4}$ from bottom to top) at $S(2-10 \mathrm{keV})=10^{-13} \mathrm{erg} \mathrm{cm}^{-2} \mathrm{~s}^{-1}$ and $S_{\nu}(0.85 \mathrm{~mm})=50 \mathrm{mJy}$ are plotted, respectively. The $\sigma_{8}$ values derived from the COBE 4 year data (Bunn \& White 1997) are also given for reference. Panels (a) and (b) show that the contours for the $2-10 \mathrm{keV}$ band counts run almost parallel

to the $\chi^{2}$ contour of the $0.5-2 \mathrm{keV}$ band counts. In this sense, future $\log N-\log S$ data in the hard X-ray band will provide an independent consistency check of the ROSAT soft X-ray data. The shape of the submm $\log N-\log S$ contours, on the other hand, is quite different, especially at high $\sigma_{8}$, and thus should place complementary constraints on $\Omega_{0}$ and $\sigma_{8}$. If the fixed shape parameter $(\Gamma=0.25)$ is adopted, the corresponding $\log N-\log S$ contours are similar to the conventional CDM $\left(\Gamma \simeq \Omega_{0} h\right)$ case, except at $\Omega_{0} \lesssim 0.2$ and $\Omega_{0} \gtrsim 0.8$. By contrast, the $C O B E$ normalized $\sigma_{8}$ is very sensitive to any changes in the spectral shape.

\section{SEARCH FOR THE SUBMM SZ EFFECT OF RXJ 1347-1195}

RXJ 1347.5-1145 $(z=0.45)$ is the most luminous X-ray cluster of galaxies known so far (Schindler et al. 1997). Its exceptionally high column density of electrons at the center implies that this is one of the most promising clusters for the detection of the first unambiguous SZ increment signal. This is clearly illustrated in Table 2 where we compute the expected central SZ intensity $\Delta I_{\nu}(0)$ at $0.85 \mathrm{~mm}$ for several clusters with measured SZ temperature decrement in the Rayleigh - Jeans regime (except for our current target, RXJ 1347-1195). Wherever available, $1 \sigma$ errors in the parameters are shown which are also adopted to estimate the error in $\Delta I_{\nu}(0)$.

Table 2: Expected central SZ intensity $\Delta I_{\nu}(0)$ at $0.85 \mathrm{~mm}$ for several clusters

\begin{tabular}{c|c|c|c|c|c|c} 
cluster & $z$ & $T[\mathrm{keV}]$ & $\beta$ & $\theta_{c}[\mathrm{arcsec}]$ & $n_{\mathrm{e}}^{0}\left[10^{-2} h_{50}^{1 / 2} \mathrm{~cm}^{-3}\right]$ & $\Delta I_{\nu}(0)\left[h_{50}^{-1 / 2} \mathrm{mJy} / \mathrm{beam}\right]$ \\
\hline RXJ 1347-1145 & 0.45 & $9.3_{-1.0}^{+1.1}$ & $0.56 \pm 0.04$ & $8.4 \pm 1.8$ & 9.4 & $7.1 \pm 1.7$ \\
\hline CL0016+16 & 0.555 & $8.4_{-0.6}^{+1.2}$ & 0.81 & 51 & 0.65 & $1.9_{-0.1}^{+0.3}$ \\
A2163 & 0.201 & $14.6 \pm 0.5$ & $0.62 \pm 0.01$ & $72 \pm 3$ & $0.77 \pm 0.03$ & $4.5 \pm 0.3$ \\
A2218 & 0.171 & $6.7 \pm 0.45$ & $0.65_{-0.03}^{+0.05}$ & $60 \pm 12$ & 0.63 & $1.2 \pm 0.3$ \\
A2142 & 0.0899 & $8.68 \pm 0.12$ & $1.0 \pm 0.3$ & $221 \pm 84$ & $0.493 \pm 0.023$ & $1.6 \pm 0.8$ \\
A478 & 0.0881 & $6.56 \pm 0.09$ & $0.67 \pm 0.03$ & $116 \pm 18$ & $0.675 \pm 0.124$ & $1.3 \pm 0.3$ \\
A2256 & 0.0581 & $7.51 \pm 0.11$ & $0.80 \pm 0.02$ & $320 \pm 12$ & $0.251 \pm 0.013$ & $0.88 \pm 0.06$ \\
Coma & 0.0235 & $9.10 \pm 0.40$ & $0.75 \pm 0.03$ & $630 \pm 36$ & $0.289 \pm 0.004$ & $1.1 \pm 0.1$
\end{tabular}

On the basis of this analysis, we performed the search for the SZ effect towards the cluster RXJ13471145, at 21 and $43 \mathrm{GHz}$ with NRO (Nobeyama Radio Observatory) 45m telescope between March 3 and 15, 1998, and at $350 \mathrm{GHz}$ with SCUBA installed on JCMT (J.C.Maxwell Telescope) 15m telescope on May 30 and 31, 1998. Although there have been a few claims of the detection of the SZ temperature increment for some clusters (e.g. Andreani et al. 1996; Lamarre et al. 1998), they measured the total flux alone which could be significantly contaminated by the dust in our Galaxy and also by submm sources in the field. Therefore it is essential to map the profile of a cluster so that one can test it against the predicted SZ signal profile. This is the reason why we attempted the observation with SCUBA which has a reasonable angular resolution, $15^{\prime \prime}$ in FWHM, with a FOV of $160^{\prime \prime}$.

As a matter of fact, it turned out that a radio point source exists near the center of our target cluster. The flux of the central point source had been determined to be $47.6 \pm 0.45 \mathrm{mJy}$ at $1.4 \mathrm{GHz}$ with VLA (Condon et al. 1988), and $11.0 \pm 0.7 \mathrm{mJy}$ at $28.5 \mathrm{GHz}$ in the OVRO serendipitous survey of SZ effect (J.Carlstrom, private communication). We also observed the central source at $100 \mathrm{GHz}$ with Nobeyama Millimeter Array (NMA) between May 19th to 21st, 1998, and found the flux to be $5.0 \pm 1.5 \mathrm{mJy}$. The 
three data at $\nu \leq 100 \mathrm{GHz}$ are very well approximated by a single power-law:

$$
F_{\mathrm{p}}(\nu)=(56.1 \pm 1.0)(\nu / 1 \mathrm{GHz})^{-0.49 \pm 0.02} \mathrm{mJy},
$$

where the quoted errors are $1 \sigma$. We subtracted the contribution of the point source according to equation (12) from our data at 21 and $43 \mathrm{GHz}$. Although the point source might be a variable, the degree of variability is known to be fairly small for radio sources with steep spectrum (spectral index $\lesssim-0.5$; e.g. Eckart, Hummel \& Witzel 1989).

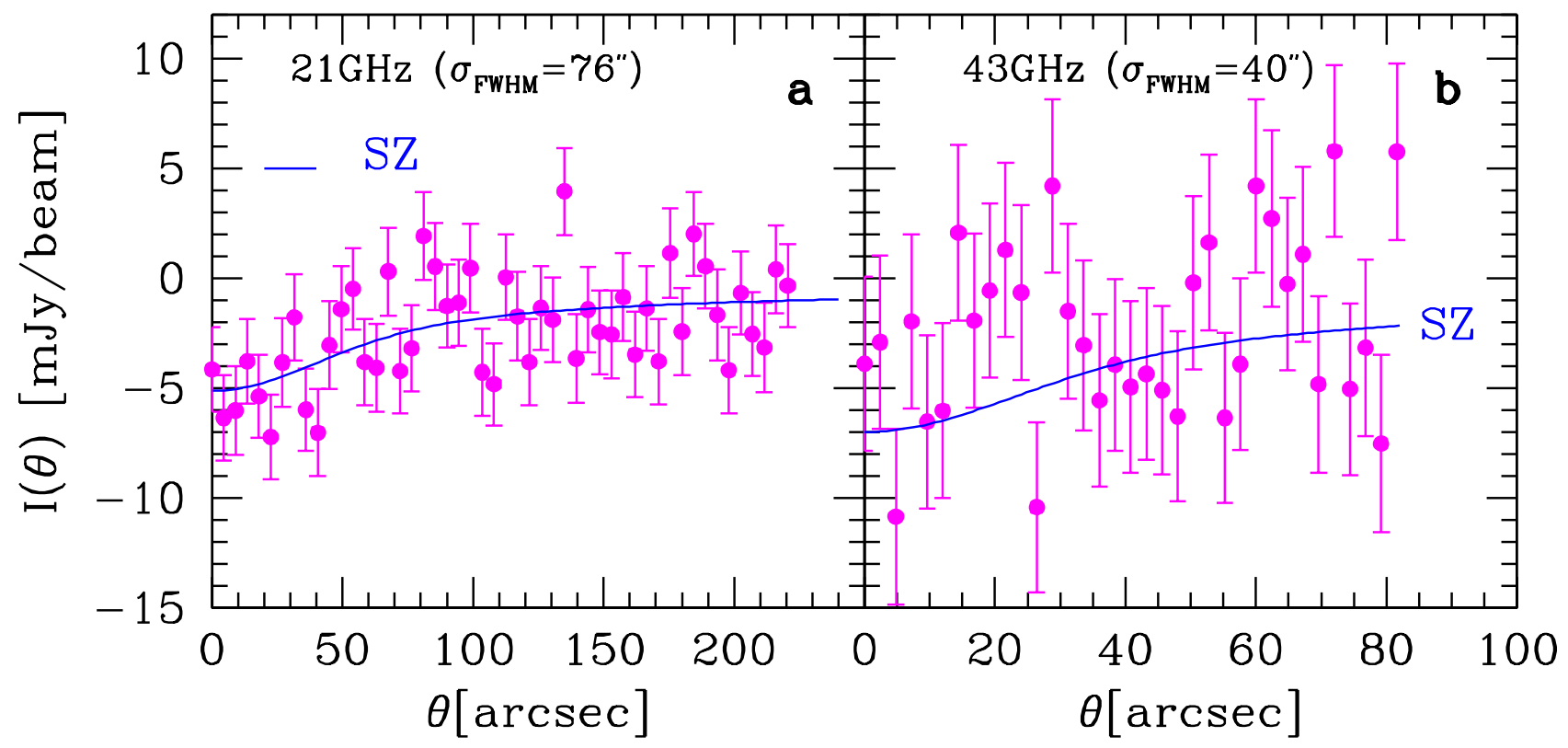

Figure 4: Radial intensity profile towards RXJ1347 at (a) 21 and (b) 43 GHz observed at NRO. The estimated contribution of the central radio source $(12.7 \mathrm{mJy}$ at $21 \mathrm{GHz}$, and $8.9 \mathrm{mJy}$ at $43 \mathrm{GHz})$ is subtracted. Filled circles indicate our data with $1 \sigma$ error-bars. Solid lines indicate the prediction of the SZ signal (without the point source contribution) using the best-fit parameters in the X-ray observation.

Figure 4 shows the profiles of the scans towards the cluster at 21 and $43 \mathrm{GHz}$ after subtracting the point source. In both cases, a extended negative intensity profile is clearly visible. Especially at $21 \mathrm{GHz}$ where the $\mathrm{S} / \mathrm{N}$ is significantly higher than at $43 \mathrm{GHz}$, the profile is quite consistent with the $\mathrm{SZ}$ profile expected from the X-ray observation. This is the first detection of the SZ temperature decrement towards the cluster RXJ 1347-1145.

The observation of the cluster at $350 \mathrm{GHz}$ with SCUBA was performed on May 30 and 31, 1998. The raw data were processed with REMSKY in SURF package to remove spatially correlated sky-noise. Since REMSKY systematically changes the base-level of the map up to the sky-noise level, the resulting zerolevel is uncertain depending on the sky condition. We estimated $1 \sigma$ error of our base-level or DC offset, $I_{\mathrm{DC}}$, to be as large as $2.9 \mathrm{mJy} /$ beam; the sky condition during our observation was not so good (the zenith optical depth at $350 \mathrm{GHz}$ ranged around $\left.\tau_{350}=0.46-0.60\right)$. Also we extracted 7 spurious contamination sources above a threshold of $3 \sigma$ using SExtractor package. The radially averaged profile of the cluster at $350 \mathrm{GHz}$ after subtracting the sources and the uncertain DC level is plotted in Figure 5. Also plotted in the figure are the SZ intensity profiles at $350 \mathrm{GHz}$ predicted from the latest X-ray observation with the point source of $F_{\mathrm{p}}=0$ and $3.2 \mathrm{mJy}$, as well as the point spread function of the $3.2 \mathrm{mJy}$ source. Since normal radio sources often show a steep decline of flux at higher (submillimeter), we regard the value, 3.2 mJy, extrapolated from equation (12) as the upper limit of the flux. Again it is clear that the extended feature of the observed intensity profile cannot be described by the point spread function alone. 


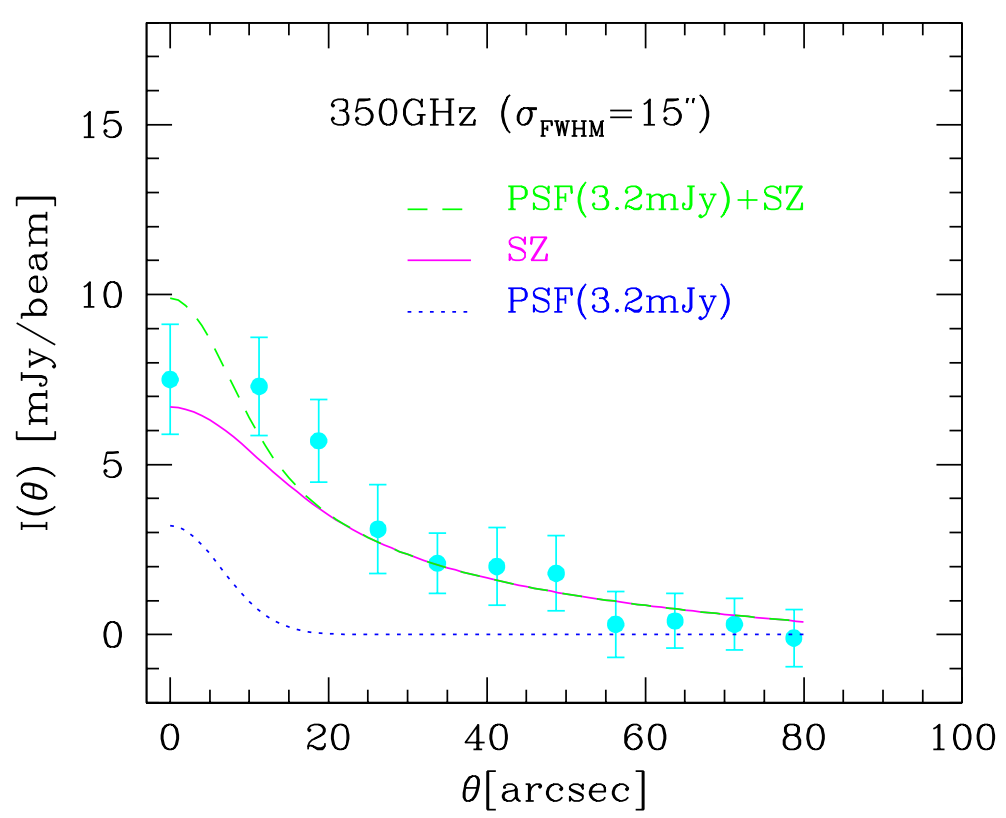

Figure 5: Radial intensity profile towards RXJ1347 at $350 \mathrm{GHz}$ observed at JCMT/SCUBA. Filled circles indicate our data with $1 \sigma$ error-bars. Dotted curve shows the PSF of $3.2 \mathrm{mJy}$ source, and dashed (solid) curves plot the SZ profiles with (without) the possible point source contribution, respectively, using the best-fit parameters in the X-ray observation.

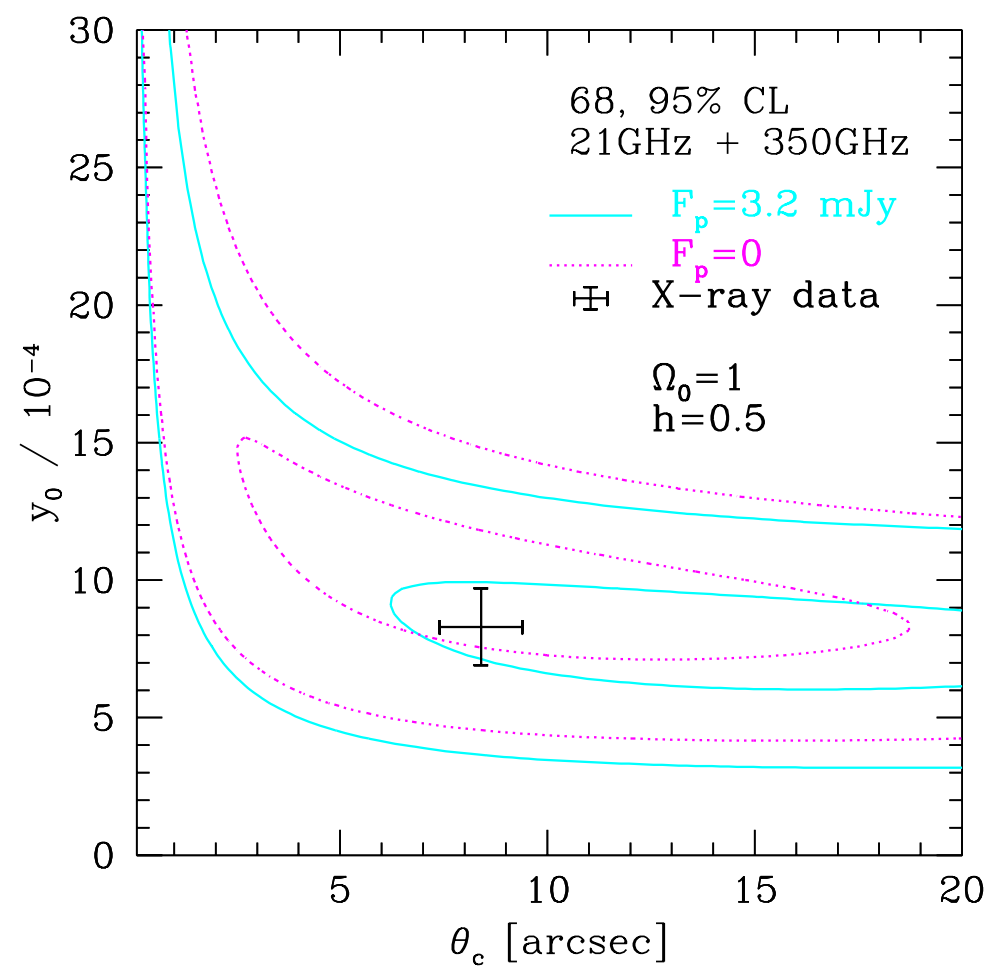

Figure 6: Confidence contours on the $y$-parameter and the core radius $\theta_{c}$ from combined data analysis at 21 and $350 \mathrm{GHz}$. The cross indicates the parameters determined from X-ray observations by ROSAT and ASCA satellites. 
To express the result in a statistical and quantitative manner, we carried out a $\chi^{2}$ fit to the SZ profiles at 21 and $350 \mathrm{GHz}$ simultaneously on the basis of the $\beta$-model with taking a central $y$-parameter, $y_{\mathrm{c}}$, and the angular core radius, $\theta_{c}$, as free parameters. The results are summarized in Figure 6 . The agreement with the X-ray observation is remarkable and reassuring, and we conclude that the present data provide the strongest and most convincing case for the detection of the submm SZ signal from the cluster. Detailed analysis taking account of various astrophysical effects and the cosmological implications will be presented elsewhere (Komatsu et al. 1999a,b).

\section{DISCUSSION AND CONCLUSIONS}

It is widely recognized now that many cosmological models are more or less successful in reproducing the structure at redshift $z \sim 0$ by construction. This is because the models have still several degrees of freedom or cosmological parameters which can be appropriately adjusted to the observations at $z \sim 0\left(\Omega_{0}\right.$, $\left.\sigma_{8}, h, \lambda_{0}, b(r, z)\right)$. There are several ways to break such cosmological degeneracy. For instance, combining the COBE 4 year data with the cluster $\log N-\log S$ observation, we showed that low-density CDM models with $\left(\Omega_{0}, \lambda_{0}, h, \sigma_{8}\right)=(0.3,0.7,0.7,1)$ and $(0.45,0,0.7,0.8)$ are viable models, while the Einstein - de Sitter model is strongly disfavored even if equation (5) can be satisfied by adjusting $\sigma_{8}$ accordingly.

Along with such consideration, we proposed a cluster survey based on the SZ effect. As a first step toward the goal, we conducted the search for the SZ effect towards the cluster RXJ1347-1145 in multi-bands. We detected the extended signal which are in good agreement with the predicted SZ profiles, especially at 21 and $350 \mathrm{GHz}$. In particular, our results provide the strongest and most convincing case for the detection of the SZ temperature increment in the submm band in which the profile of a cluster of galaxies is resolved for the first time.

We thank Iain Coulson, Nario Kuno and Satoki Matsushita for kind assistance during our observing runs at JCMT, Nobeyama 45-m and Nobeyama Millimeter Array, respectively. We also thank Nick Tothill for providing the calibration data for our observation, and Tim Jenness, John Richer, Remo Tilanus and Goeran Sandell for many fruitful comments and suggestions on data analysis via the SCUBADR mailing list. We are also grateful to John Carlstrom for information of the flux of the central source at 28.5 GHz. The travel of E. K. to Hawaii was supported in part by Satio Hayakawa Foundation in the Astronomical Society of Japan. T. K. gratefully acknowledges support from a JSPS (Japan Society for the Promotion of Science) fellowship. This research was supported in part by the Grants-in-Aid for the Center-of-Excellence (COE) Research of the Ministry of Education, Science, Sports and Culture of Japan to RESCEU (No.07CE2002). 


\section{REFERENCES}

Andreani, P., et al. Astrophys.J., 459, L49 (1996)

Bryan, G. L., Norman, M. L. Astrophys.J., 495, 80 (1998)

Bunn, E. F., White, M. Astrophys.J., 480, 6 (1997)

Cagnoni, I., Della Ceca, R., Maccacaro, T. Astrophys.J., 493, 54 (1998)

Condon, J. J. et al. Astron. J., 115, 1693 (1998)

Ebeling, H., Edge, A. C., Fabian, A.C., Allen, S. W., Crawford, C. S. Astrophys. J., 479, L101 (1997)

Ebeling H., et al. Mon.Not.R.Astron.Soc., submitted (1998)

Eckart, A., Hummel, C. A., Witzel, A. Mon. Not. R. Astron. Soc., 239, 381 (1989).

David, L. P., Slyz, A., Jones, C., Forman, W., Vrtilek, S. D. Astrophys. J., 412, 479 (1993)

Kitayama, T., Sasaki, S., Suto, Y. Publ. Astron. Soc. Japan, 50, 1 (1998)

Kitayama, T., Suto, Y. Mon.Not.R.Astron.Soc., 280, 638 (1996a)

Kitayama, T., Suto, Y., Astrophys.J., 469, 480 (1996b).

Kitayama, T., Suto, Y., Astrophys.J., 490, 557 (1997).

Komatsu, E., Kitayama, T., Suto, Y., Hattori, M., Kawabe, R., Matsuo, H., Schindler, S., Yoshikawa, K., Astrophys.J., in press (1999a)

Komatsu, E., et al., in preparation (1999b)

Lacey, C. G., Cole, S. Mon.Not.R.Astron.Soc., 262, 627 (1993)

Lamarre, J. M., et al. Astrophys. J. 507, L5 (1998)

Mushotzky, R. F., Scharf, C. A. Astrophys.J., 482, L13 (1997)

Piccinotti, G., Mushotzky, R. F., Boldt, E. A., Holt, S. S., Marshall, F. E., Serlemitsos, P. J., Shafer, R. A. Astrophys.J., 253, 485 (1982)

Press, W. H., Schechter, P. Astrophys.J., 187, 425 (1974)

Rephaeli, Y. ARA\&A 33, 541 (1995)

Rephaeli, Y., Yankovitch, D. Astrophys.J., 481, L55 (1997)

Rosati, P., Della Ceca, R., Burg R., Norman, C., \& Giacconi, R. Astrophys.J., 445, L11 (1995)

Rosati, P., Della Ceca, R., Norman, C., Giacconi, R. Astrophys. J., 492, L21 (1998)

Sasaki, S. Publ. Astron. Soc. Japan, 46, 427 (1994)

Schindler, S., Hattori, M., Neumann, D. M. Böhringer, H. Astron. Astrophys., 317, 646 (1997)

Sunyaev R.A., Zel'dovich Ya.B. Commts. Astrophys. Space Phys., 4, 173 (1972)

Ueda, Y., Takahashi, T., Inoue, H., Tsuru, T., Sakano, M., Ishisaki, Y., Ogasaka, Y., Makishima, K., Yamada, T., Ohta, K., Akiyama, M. Nature, 391, 866 (1998) 